\title{
Cyclic and finite surgeries on Montesinos knots
}

\author{
KAZUHIRO ICHIHARA \\ IN DAE JONG
}

\begin{abstract}
We give a complete classification of the Dehn surgeries on Montesinos knots which yield manifolds with cyclic or finite fundamental groups.
\end{abstract}

57M50; 57M25

\section{Introduction}

A Dehn surgery on a knot $K$ in a 3 -manifold $M$ is an operation to create a new 3-manifold from $M$ and $K$ as follows: Remove an open tubular neighborhood of $K$, and glue a solid torus back. By gluing a solid torus back as it was, the surgery gives the original manifold again. So such a surgery is called trivial, and we will ignore it in general.

On knots in the 3 -sphere $S^{3}$, it is an interesting problem to determine and classify all nontrivial Dehn surgeries which produce 3-manifolds with cyclic or finite fundamental groups, which we call cyclic surgeries or finite surgeries, respectively.

As part of the Hyperbolic Dehn Surgery Theorem, Thurston [23] established that there are finitely many cyclic and finite surgeries. In fact, Culler, Gordon, Luecke and Shalen [4] (respectively, Boyer and Zhang [3]) proved there are at most three cyclic (resp. five finite) surgeries. Furthermore, it is conjectured that knots admitting cyclic (resp. finite) surgeries are doubly primitive (resp. primitive/Seifert fibered) knots as introduced by Berge [1] (resp. Dean [5]). See Kirby [13, Problem 1.77] for more information.

Cyclic and finite surgeries have been studied extensively for some classes of knots. For example, it was shown by Delman and Roberts in [8] that no hyperbolic alternating knot admits a cyclic or finite surgery.

One of the other well-known classes of knots, containing nonalternating ones, is the Montesinos knots. A Montesinos knot is defined as a knot admitting a diagram obtained by putting rational tangles together in a circle. See Figure 1 for instance. In particular, 


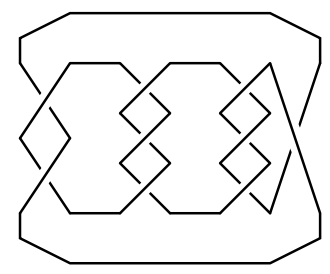

Figure 1: A diagram of a Montesinos knot

a Montesinos knot $K$ is called a $\left(a_{1}, a_{2}, \ldots, a_{n}\right)$-pretzel knot if the rational tangles in $K$ are of the form $1 / a_{1}, 1 / a_{2}, \ldots, 1 / a_{n}$.

In this paper, based on studies by Delman [6] and Mattman [15], we give a complete classification of cyclic / finite surgeries on Montesinos knots as follows.

Theorem 1.1 Let $K$ be a hyperbolic Montesinos knot. If $K$ admits a nontrivial cyclic surgery, then $K$ must be equivalent to the $(-2,3,7)$-pretzel knot and the surgery slope is 18 or 19. If $K$ admits a nontrivial acyclic finite surgery, then $K$ must be equivalent to either the $(-2,3,7)$-pretzel knot and the surgery slope is 17 , or the $(-2,3,9)-$ pretzel knot and the surgery slope is 22 or 23 .

As a direct corollary, together with the result by $\mathrm{Wu}$ [25], we have the following.

Corollary 1.2 Let $K$ be a hyperbolic arborescent knot. If $K$ admits a nontrivial cyclic surgery, then $K$ must be equivalent to the $(-2,3,7)$-pretzel knot and the surgery slope is 18 or 19. If $K$ admits a nontrivial acyclic finite surgery, then $K$ must be equivalent to either the $(-2,3,7)$-pretzel knot and the surgery slope is 17 , or the $(-2,3,9)-$ pretzel knot and the surgery slope is 22 or 23.

Recently, using Khovanov homology, it was shown by Watson in [24, Theorem 7.5] that the $(-2, p, p)$-pretzel knot does not admit finite surgeries for $p \in\{5,7, \ldots, 25\}$.

Very recently, Futer, Ishikawa, Kabaya, Mattman and Shimokawa [9] obtained, independently, a complete classification of finite surgeries on $(-2, p, q)$-pretzel knots with odd positive integers $p$ and $q$.

Remark 1 It is already known which Montesinos knots are nonhyperbolic. If a Montesinos knot is equivalent to one consisting of at most two rational tangles, then it actually is a two-bridge knot. Menasco [16] showed that the nonhyperbolic twobridge knots are the $(2, p)$-torus knots. The only other nontrivial nonhyperbolic Montesinos knots are the $(-2,3,3)-$ and $(-2,3,5)$-pretzel knots, which are actually 
the $(3,4)$ - and $(3,5)$-torus knots, respectively. This was originally shown by Oertel [ 19 , Corollary 5] as well as in an unpublished monograph [2] by Bonahon and Siebenmann. The cyclic and finite surgeries of torus knots have been completely classified by Moser [17].

To prove Theorem 1.1, we will prepare two propositions, Proposition 2.1 and Proposition 3.1, which will be shown in Section 2 and Section 3 respectively. Then, in the last section, Theorem 1.1 will be proved from these propositions together with a result of Mattman [15].

Acknowledgments The authors would like to thank Mikami Hirasawa for useful conversations about the fiberedness of pretzel knots as in the proof of Claim 1. They also wish to thank Hiroshi Matsuda for his useful comments about the rank and the Euler characteristic of Heegaard Floer homology as in the proof of Claim 2, and also thank Thomas Mattman for his useful comments about cyclic and finite surgeries on arborescent knots as in Corollary 1.2. They also thank the referee for careful reading of our draft and useful suggestions to improve the proof of Claim 2.

The first author is partially supported by Grant-in-Aid for Young Scientists (B), No. 20740039, Ministry of Education, Culture, Sports, Science and Technology, Japan.

\section{Cyclic/finite surgeries and the Alexander polynomials}

In this section, we prove the following proposition.

Proposition 2.1 Let $K$ be a hyperbolic Montesinos knot admitting a nontrivial cyclic or finite surgery. Then $K$ is equivalent to a $(-1,2 n, p, q)$-pretzel knot, where $n$ is a nonzero integer and $p, q$ are odd positive integers with $3 \leq p \leq q$. Furthermore all nonzero coefficients of the Alexander polynomial for $K$ are \pm 1 .

Proof Suppose that a hyperbolic Montesinos knot $K$ admits a nontrivial cyclic or finite surgery. Then Delman showed in $[7 ; 6]$ that $K$ must be equivalent to either a $(-2 l, p, q)$-pretzel knot, a $(-1,2 n, p, q)$-pretzel knot or a $(-1,-1,2 m, p, q)$-pretzel knot with an integer $n$, integers $l, m>1$ and odd positive integers $p, q(3 \leq p \leq q)$. Also see a survey [26, Section 2, Section 3] by Wu. Actually Delman showed that any Montesinos knot except for those listed above admits an essential lamination in its exterior which survives all nontrivial Dehn surgeries. Essential laminations were introduced by Gabai and Oertel in [11] and, actually, they showed that if a 3-manifold admits an essential lamination, then its universal cover must be the 3 -space $\mathbb{R}^{3}$. In 
particular its fundamental group is not cyclic or finite. See Gabai and Oertel [11] for the precise definition.

By virtue of Delman's result, in order to prove Proposition 2.1, it suffices to show that the first and the third types of pretzel knots described above cannot have cyclic or finite surgeries. Note here that a $(-2, p, q)$-pretzel knot (the case $l=1$ in the first) is equivalent to a $(-1,2, p, q)$-pretzel knot (the case $n=1$ in the second). Also a $(-1,-1,2, p, q)$-pretzel knot (the case $m=1$ in the third) is equivalent to a $(-1,-2, p, q)$-pretzel knot (the case $n=-1$ in the second). Thus, excluding over laps, we are assuming $l, m \neq 1$.

Among the classes of knots described above, the first one was already studied by Mattman in [15]. He actually showed in [15, Theorem 1.1 and 1.2] that any $(-2 l, p, q)-$ pretzel knot with $l>1$ and odd positive integers $p, q(3 \leq p \leq q)$ has neither cyclic surgeries nor finite surgeries.

Thus, in the following, we focus on the third class above. We here use the following strong result of $\mathrm{Ni}$ [18, Corollary 1.3], established by using the Heegaard Floer homology theory: If a knot in $S^{3}$ admits a cyclic or finite surgery, then it must be a fibered knot. Actually he showed that a knot $K$ in $S^{3}$ must be fibered if $K$ admits a surgery yielding an L-space. Here a rational homology sphere $Y$ is called an $L$-space if the rank of $\widehat{H F}(Y)$ is equal to $\left|H_{1}(Y ; \mathbb{Z})\right|$. In fact, any 3 -manifold with a cyclic or finite fundamental group is an L-space, as is shown by Ozsváth and Szabó in [22, Proposition 2.3].

Now, the next claim, together with the result by $\mathrm{Ni}$, imply the first conclusion of Proposition 2.1.

Claim 1 Let $m>1$ and $p, q$ be odd positive integers where $p \leq q$. Then the $(-1,-1,2 m, p, q)$-pretzel knot is not fibered.

Proof We just apply the algorithm given by Gabai in [10, Theorem 6.7]. Here we include only an outline, assuming that the reader is rather familiar with [10, Theorem 6.7].

Let $K$ be a $(-1,-1,2 m, p, q)$-pretzel knot with an integer $m>1$ and odd positive integers $p, q(p \leq q)$. We start to apply the algorithm in [10, Theorem 6.7] with $n_{1}=-1, n_{2}=-1, n_{3}=2 m, n_{4}=p, n_{5}=q$. After a cyclic permutation, the surface $R$ obtained by applying Seifert's algorithm is of type II in [10, TYPE II.6.5] with $m_{1}=-1$, $m_{11}=2 m, m_{2}=p, m_{3}=q, m_{4}=-1$. (See [10, Figure 6.3].) We now see CASE 2 in [10, Theorem 6.7]. Here we note that the associated oriented pretzel link $L^{\prime}$ (defined in $\left[10\right.$, TYPE II.6.5]) is of type $(2 m,-2,-2)$. Since $\sum_{j=1}^{4} m_{j} /\left|m_{j}\right|=-1+1+1-1=0$ 
and $L^{\prime}$ is of type $(2 m,-2,-2) \neq \pm(2,-2,2)$ if $m>1$, we check CASE $2 \mathrm{~B}$ in [10, Theorem 6.7]. Then we see that $K$ is fibered if and only if $L^{\prime}$ is fibered. For $L^{\prime}$, we check CASE 1 in [10, Theorem 6.7], and verify that $L^{\prime}$ is not fibered since no $n_{j}$ is \pm 1 and $L^{\prime}$ is not equivalent to a pretzel link of type $\pm(2,-2, \ldots, 2,-2, n)$ with an integer $n$. Therefore we conclude that $K$ is not fibered.

The second conclusion of Proposition 2.1 follows from results of Ozsvath and Szabó, also achieved by using the Heegaard Floer homology theory in $[21 ; 22 ; 20]$. We first prepare the following claim, which is implicitly used in [18, Proof of Corollary 1.3].

Claim 2 If $\alpha / \beta$-Dehn surgery on a nontrivial knot $K$ in $S^{3}$ yields an $L$-space for some coprime integers $\alpha, \beta$ with $\beta \geq 2$, then $\alpha$-Dehn surgery on $K$ also yields an $L-$ space.

Proof Given coprime integers $\alpha, \beta$ and a knot $K$ in $S^{3}$, let $S_{\alpha / \beta}^{3}(K)$ denote the 3-manifold obtained from $S^{3}$ by $\alpha / \beta$-Dehn surgery on $K$. We recall the following general formula given in [20, Proposition 9.5]:

$\operatorname{rk} \widehat{H F}\left(S_{\alpha / \beta}^{3}(K)\right)=|\alpha|+2 \max (0,(2 v(K)-1)|\beta|-|\alpha|)+|\beta|\left(\sum_{s}\left(\operatorname{rk} H_{*}\left(\widehat{A_{S}}\right)-1\right)\right)$.

This holds for any pair of coprime integers $\alpha, \beta$.

For simplicity, let $X(v(K), \alpha, \beta)$ denote $\max (0,(2 v(K)-1)|\beta|-|\alpha|)$ and $Y$ denote $\sum_{s}\left(\operatorname{rk} H_{*}\left(\widehat{A}_{S}\right)-1\right)$. Then we have

$$
\text { rk } \widehat{H F}\left(S_{\alpha / \beta}^{3}(K)\right)=|\alpha|+2 X(v(K), \alpha, \beta)+|\beta| Y .
$$

Now, for some coprime integers $\alpha, \beta$ with $\beta \geq 2$, we assume that $S_{\alpha / \beta}^{3}(K)$ is an L-space, ie, by definition,

$$
\operatorname{rk} \widehat{H F}\left(S_{\alpha / \beta}^{3}(K)\right)=|\alpha| .
$$

It then suffices to show that $S_{\alpha}^{3}(K)$ is an L-space, ie, $\operatorname{rk} \widehat{H F}\left(S_{\alpha}^{3}(K)\right)=|\alpha|$.

On the other hand, in general, we see that $\operatorname{rk} \widehat{H F}\left(S_{\alpha}^{3}(K)\right)-|\alpha| \geq 0$ for any integer $\alpha$ as follows. In the proof of [21, Proposition 5.1], it is claimed that

$$
\chi\left(\widehat{H F}\left(S_{\alpha}^{3}(K)\right)\right)=\left|H_{1}\left(S_{\alpha}^{3}(K) ; \mathbb{Z}\right)\right| .
$$

Also see [22, Section 2]. By definition, the Euler characteristic (the left-hand side) is the alternating sum of the dimensions of $\widehat{H F}\left(S_{\alpha}^{3}(K)\right)$. Hence, it is not greater than the total rank of $\widehat{H F}\left(S_{\alpha}^{3}(K)\right)$, ie,

$$
\operatorname{rk} \widehat{H F}\left(S_{\alpha}^{3}(K)\right) \geq \chi\left(\widehat{H F}\left(S_{\alpha}^{3}(K)\right)\right)=\left|H_{1}\left(S_{\alpha}^{3}(K) ; \mathbb{Z}\right)\right|=|\alpha| .
$$


From this equation, in order to obtain $\operatorname{rk} \widehat{H F}\left(S_{\alpha}^{3}(K)\right)=|\alpha|$, it suffices to show that $\operatorname{rk} \widehat{H F}\left(S_{\alpha}^{3}(K)\right)-|\alpha| \leq 0$. Actually, we have from Equation (1)

$$
\text { rk } \widehat{H F}\left(S_{\alpha}^{3}(K)\right)-|\alpha|=2 X(v(K), \alpha, 1)+Y .
$$

Note here that we have $Y \leq 0$ as follows. It is seen that

$$
2 X(v(K), \alpha, \beta)+|\beta| Y=0
$$

from Equation (1) and the assumption that $\operatorname{rk} \widehat{H F}\left(S_{\alpha / \beta}^{3}(K)\right)=|\alpha|$. Thus, together with $X(v(K), \alpha, \beta) \geq 0$ by definition, we have $Y \leq 0$.

If $v(K) \leq 0$, then

$$
X(v(K), \alpha, 1)=\max (0,(2 v(K)-1)-|\alpha|)=0 .
$$

Since $Y \leq 0$, together with Equation (2), we obtain that $\operatorname{rk} \widehat{H F}\left(S_{\alpha}^{3}(K)\right)-|\alpha| \leq 0$ as desired.

If $v(K) \geq 1$, then we have $X(v(K), \alpha, 1)<X(v(K), \alpha, \beta)$ from the assumption that $\beta \geq 2$. Thus, together with $Y \leq 0$ and Equation (3), we obtain that

$$
2 X(v(K), \alpha, 1)+Y<2 X(v(K), \alpha, \beta)+Y=-|\beta| Y+Y \leq 0 .
$$

Together with Equation (2), this implies that $\operatorname{rk} \widehat{H F}\left(S_{\alpha}^{3}(K)\right)-|\alpha| \leq 0$ as desired.

Then, in [22, Corollary 1.3], Ozsváth and Szabó proved that if a knot $K$ in $S^{3}$ admits an integral Dehn surgery yielding an L-space, then the Alexander polynomial $\Delta_{K}(t)$ has the form

$$
\Delta_{K}(t)=(-1)^{k}+\sum_{j=1}^{k}(-1)^{k-j}\left(t^{n_{j}}+t^{-n_{j}}\right)
$$

for some increasing sequence of positive integers $0<n_{1}<n_{2}<\cdots<n_{k}$. This means that all nonzero coefficients of $\Delta_{K}(t)$ are \pm 1 .

Remark 2 In the above proof, Claim 2 is actually necessary for the following reason. By the Cyclic Surgery Theorem established in [4], all cyclic surgeries on hyperbolic knots in $S^{3}$ are shown to be integral surgeries. However, the Finite Surgery Theorem of [3] shows that finite surgeries on hyperbolic knots in $S^{3}$ are half-integral or integral. In other words, at present, we cannot rule out the possibility of a half-integral surgery and it is currently only a conjecture that such finite surgeries are integral: See Kirby [13, Problem 1.77 A(6)] for more information. 


\section{Calculation of the Alexander polynomials}

In this section, we prove the following proposition, which will be shown by direct calculations of the Alexander polynomials.

Proposition 3.1 Let $K$ be a pretzel knot of type $(-1,2 n, p, q)$, where $n$ is an integer and $p, q$ are odd positive integers with $p \leq q$. If every nonzero coefficient of the Alexander polynomial of $K$ is \pm 1 , then $n=1$ and $p=3$.

Recall that the Alexander polynomial $\Delta_{L}(t)$ for a link $L$ satisfies the following skein relation (see Lickorish [14, page 82] for example):

$$
\Delta_{L_{+}}(t)-\Delta_{L_{-}}(t)=\left(t^{-1 / 2}-t^{1 / 2}\right) \Delta_{L_{0}}(t),
$$

where $L_{+}, L_{-}$, and $L_{0}$ possess diagrams $D_{+}, D_{-}$and $D_{0}$ which differ only in a small neighborhood as shown in Figure 2.
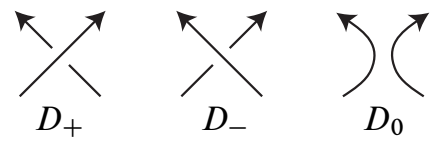

Figure 2: Skein triples

Remark 3 Let $l$ be a positive integer, and $\Delta_{l}(t)$ the Alexander polynomial of a $(2, l)$-torus link. Set $f_{l}=\sum_{i=0}^{l} t^{i}$. Then we have $\Delta_{l}(-t)=(-t)^{(1-l) / 2} f_{l-1}$. See Kawauchi [12, page 98] for example.

Proof of Proposition 3.1 We divide our proof of Proposition 3.1 into three claims. We denote by $P\left(a_{1}, \ldots, a_{j}\right)$ a pretzel link of type $\left(a_{1}, \ldots, a_{j}\right)$, and by $[g(t)]_{j}$ the coefficient of $t^{j}$ in a polynomial $g(t)$.

Claim 3 Let $n$ be an integer with $n \geq 1$. Let $p$ and $q$ be odd integers with $3 \leq p \leq q$. Let $K$ be a pretzel knot of type $(-1,-2 n, p, q)$. Then we have

$$
\left[\Delta_{K}(t)\right]_{1}=\left\{\begin{array}{l}
-4 \text { if } n=1, \\
-3 \text { if } n \geq 2,
\end{array}\right.
$$

where $\Delta_{K}(t)$ is normalized so that mindeg $\Delta_{K}(t)=0$ and $\left[\Delta_{K}(t)\right]_{0}>0$.

Proof Let $K=P(-1,-2 n, p, q)$ with $1 \leq n$ and $3 \leq p \leq q$. By applying the skein formula (4) at crossings in the $(-2 n)$-twists repeatedly, we can obtain a resolving tree such that each leaf node corresponds to either $P(-1,0, p, q)$ or $P(-1,-1, p, q)$. 
Notice that $P(-1,0, p, q)$ is equivalent to a connected sum of a $(2, p)$-torus knot and a $(2, q)$-torus knot. Then we have

$$
\begin{aligned}
\Delta_{K}(t) & =\Delta_{2 n-1}(t) \Delta_{P(-1,0, p, q)}(t)-\Delta_{2 n}(t) \Delta_{P(-1,-1, p, q)}(t) \\
& =\Delta_{2 n-1}(t) \Delta_{p}(t) \Delta_{q}(t)-\Delta_{2 n}(t) \Delta_{P(-1,-1, p, q)}(t) .
\end{aligned}
$$

Next we calculate the Alexander polynomial of $P(-1,-1, p, q)$ by the same argument as above. By applying the skein formula (4) at crossings in the $p$-twists repeatedly, we can obtain a resolving tree such that each leaf node corresponds to either $P(-1,-1,0, q)$ or $P(-1,-1,1, q)$. Note that $P(-1,-1,0, q)$ is equivalent to a $(2, q)$-torus knot and that $P(-1,-1,1, q)$ is equivalent to a $(2, q-1)$-torus link. Then we have

$$
\begin{aligned}
\Delta_{P(-1,-1, p, q)}(t) & =\Delta_{p-1}(t) \Delta_{P(-1,-1,0, q)}(t)+\Delta_{p}(t) \Delta_{P(-1,-1,1, q)}(t) \\
& =\Delta_{p-1}(t) \Delta_{q}(t)+\Delta_{p}(t) \Delta_{q-1}(t) .
\end{aligned}
$$

Hence we have

$$
\Delta_{K}(t)=\Delta_{2 n-1}(t) \Delta_{p}(t) \Delta_{q}(t)-\Delta_{2 n}(t) \Delta_{p-1}(t) \Delta_{q}(t)-\Delta_{2 n}(t) \Delta_{p}(t) \Delta_{q-1}(t) .
$$

To calculate easily, we consider the polynomial obtained by substituting $-t$ in the Alexander polynomial, namely, $\Delta_{K}(-t)$. By Remark 3, we have

$$
\begin{aligned}
\Delta_{K}(-t) & =(-t)^{(4-p-q-2 n) / 2}\left(f_{2 n-2} f_{p-1} f_{q-1}-f_{2 n-1} f_{p-2} f_{q-1}-f_{2 n-1} f_{p-1} f_{q-2}\right) \\
& \doteq-f_{2 n-2} f_{p-1} f_{q-1}+f_{2 n-1} f_{p-2} f_{q-1}+f_{2 n-1} f_{p-1} f_{q-2} .
\end{aligned}
$$

Here the symbol $\doteq$ means that both sides are equal up to multiplications by units of the Laurent polynomial ring $\mathbb{Z}\left[t, t^{-1}\right]$. Here we recall that $1 \leq n$ and $3 \leq p \leq q$. Then we have

$$
\begin{aligned}
& {\left[f_{2 n-2} f_{p-1} f_{q-1}\right]_{1}=\left\{\begin{array}{l}
2 \text { if } n=1, \\
3 \text { if } n \geq 2,
\end{array}\right.} \\
& {\left[f_{2 n-1} f_{p-2} f_{q-1}\right]_{1}=3,} \\
& {\left[f_{2 n-1} f_{p-1} f_{q-2}\right]_{1}=3 .}
\end{aligned}
$$

Therefore we have

that is,

$$
\begin{aligned}
{\left[\Delta_{K}(-t)\right]_{1} } & =\left\{\begin{array}{l}
-2+3+3=4 \text { if } n=1, \\
-3+3+3=3 \text { if } n \geq 2,
\end{array}\right. \\
{\left[\Delta_{K}(t)\right]_{1} } & =\left\{\begin{array}{l}
-4 \text { if } n=1, \\
-3 \text { if } n \geq 2 .
\end{array}\right.
\end{aligned}
$$

This completes the proof. 
Claim 4 Let $n$ be an integer with $n \geq 2$. Let $p$ and $q$ be odd integers with $3 \leq p \leq q$. Let $K$ be a pretzel knot of type $(-1,2 n, p, q)$. Then we have

$$
\left[\Delta_{K}(t)\right]_{3}=2,
$$

where $\Delta_{K}(t)$ is normalized so that mindeg $\Delta_{K}(t)=0$ and $\left[\Delta_{K}(t)\right]_{0}>0$.

Proof The proof is similar to that of Claim 3. Let $K=P(-1,2 n, p, q)$ with $2 \leq n$ and $3 \leq p \leq q$. By applying the skein formula (4) at crossings in the $2 n$-twists repeatedly, we can obtain a resolving tree such that each leaf node corresponds to $P(-1,0, p, q)$ or $P(-1,1, p, q)$. Then we have

$$
\begin{aligned}
\Delta_{K}(t) & =\Delta_{2 n-1}(t) \Delta_{P(-1,0, p, q)}(t)+\Delta_{2 n}(t) \Delta_{P(-1,1, p, q)}(t) \\
& =\Delta_{2 n-1}(t) \Delta_{p}(t) \Delta_{q}(t)+\Delta_{2 n}(t) \Delta_{P(-1,1, p, q)}(t) .
\end{aligned}
$$

By applying the same argument as above at crossings in the $p$-twists, we have

$$
\begin{aligned}
\Delta_{P(-1,1, p, q)}(t) & =\Delta_{p-1}(t) \Delta_{P(-1,1,0, q)}(t)+\Delta_{p}(t) \Delta_{P(-1,1,1, q)}(t) \\
& =\Delta_{p-1}(t) \Delta_{q}(t)+\Delta_{p}(t) \Delta_{P(-1,1,1, q)}(t) .
\end{aligned}
$$

Notice that $P(-1,1,1, q)$ is equivalent to a $(2, q+1)$-torus link. By applying the skein formula (4), we have $\Delta_{q+1}=\Delta_{q-1}(t)+\left(t^{-1 / 2}-t^{1 / 2}\right) \Delta_{q}(t)$. Hence we have

$$
\begin{aligned}
\Delta_{K}(t)=\Delta_{2 n-1}(t) \Delta_{p}(t) \Delta_{q}(t) & +\Delta_{2 n}(t) \Delta_{p-1}(t) \Delta_{q}(t)+\Delta_{2 n}(t) \Delta_{p}(t) \Delta_{q-1}(t) \\
& +\left(t^{-1 / 2}-t^{1 / 2}\right) \Delta_{2 n}(t) \Delta_{p}(t) \Delta_{q}(t),
\end{aligned}
$$

and then we have

$$
\begin{gathered}
\Delta_{K}(-t) \doteq-t f_{2 n-2} f_{p-1} f_{q-1}-t f_{2 n-1} f_{p-2} f_{q-1}-t f_{2 n-1} f_{p-1} f_{q-2} \\
+(1+t) f_{2 n-1} f_{p-1} f_{q-1} .
\end{gathered}
$$

Here we recall that $2 \leq n$ and $3 \leq p \leq q$. Then we have

$$
\begin{aligned}
& {\left[t f_{2 n-2} f_{p-1} f_{q-1}\right]_{3}=\left[f_{2 n-2} f_{p-1} f_{q-1}\right]_{2}} \\
& =6 \text {, } \\
& {\left[t f_{2 n-1} f_{p-2} f_{q-1}\right]_{3}=\left[f_{2 n-1} f_{p-2} f_{q-1}\right]_{2}} \\
& =\left\{\begin{array}{l}
5 \text { if } p=3, q \geq 3, \\
6 \text { if } 5 \leq p \leq q,
\end{array}\right. \\
& {\left[t f_{2 n-1} f_{p-1} f_{q-2}\right]_{3}=\left[f_{2 n-1} f_{p-1} f_{q-2}\right]_{2}} \\
& =\left\{\begin{array}{l}
5 \text { if } p=3, q=3, \\
6 \text { if } p \geq 3, q \geq 5,
\end{array}\right.
\end{aligned}
$$




$$
\begin{aligned}
{\left[(1+t) f_{2 n-1} f_{p-1} f_{q-1}\right]_{3}=} & {\left[f_{2 n-1} f_{p-1} f_{q-1}\right]_{3}+\left[f_{2 n-1} f_{p-1} f_{q-1}\right]_{2} } \\
& =\left\{\begin{array}{l}
8+6=14 \text { if } p=3, q=3, \\
9+6=15 \text { if } p=3, q \geq 5, \\
10+6=16 \text { if } 5 \leq p \leq q .
\end{array}\right.
\end{aligned}
$$

Therefore we have

$$
\left[\Delta_{K}(-t)\right]_{3}=\left\{\begin{array}{l}
-6-5-5+14=-2 \text { if } p=3, q=3 \\
-6-5-6+15=-2 \text { if } p=3, q \geq 5 \\
-6-6-6+16=-2 \text { if } 5 \leq p \leq q
\end{array}\right.
$$

that is, $\left[\Delta_{K}(t)\right]_{3}=2$.

Here we note that $P(-1,2, p, q)$ is equivalent to $P(-2, p, q)$.

Claim 5 Let $p$ and $q$ be odd integers with $5 \leq p \leq q$. Let $K$ be a pretzel knot of type $(-2, p, q)$. Then we have

$$
\left[\Delta_{K}(t)\right]_{4}=-2,
$$

where $\Delta_{K}(t)$ is normalized so that mindeg $\Delta_{K}(t)=0$ and $\left[\Delta_{K}(t)\right]_{0}>0$.

Proof Let $K=P(-2, p, q)$ with $5 \leq p \leq q$. By applying the skein formula (4) at a crossing in the $(-2)-$ twists, we have

$$
\Delta_{K}(t)=\Delta_{p}(t) \Delta_{q}(t)+\left(t^{-1 / 2}-t^{1 / 2}\right) \Delta_{p+q}(t) .
$$

Then we have

$$
\Delta_{K}(-t) \doteq-t f_{p-1} f_{q-1}+(1+t) f_{p+q-1} .
$$

Here we recall that $5 \leq p \leq q$. Then we have $\left[t f_{p-1} f_{q-1}\right]_{4}=\left[f_{p-1} f_{q-1}\right]_{3}=4$ and $\left[(1+t) f_{p+q-1}\right]_{4}=1+1=2$. Therefore we have $\left[\Delta_{K}(-t)\right]_{4}=-4+2=-2$, that is, $\left[\Delta_{K}(t)\right]_{4}=-2$.

This completes the proof of Proposition 3.1.

\section{Proof of Theorem 1.1}

Proof of Theorem 1.1 By Proposition 2.1 and Proposition 3.1, if a hyperbolic Montesinos knot $K$ admits a nontrivial cyclic or finite surgery, then $K$ is equivalent to a $(-1,2,3, q)$-pretzel knot, where $q$ is an odd positive integer with $3 \leq q$. This $K$ is 
actually equivalent to a $(-2,3, q)$-pretzel knot. Then Mattman showed in [15, Theorems 1.1 and 1.2] that, among such pretzel knots, only the $(-2,3,7)-$ and $(-2,3,9)-$ can have cyclic/finite surgeries, and the surgery slopes are as described in Theorem 1.1. This completes the proof of Theorem 1.1.

Remark 4 The techniques we have used in this paper cannot be applied to the $(-2,3, q)$-pretzel knots as they are fibered and all nonzero coefficients of their Alexander polynomials are \pm 1 .

\section{References}

[1] J Berge, Some knots with surgeries yielding lens spaces, unpublished manuscript

[2] F Bonahon, L Siebenmann, Geometric splittings of knots, and Conway's algebraic knots, draft of a monograph (1979-1985)

[3] S Boyer, X Zhang, A proof of the finite filling conjecture, J. Differential Geom. 59 (2001) 87-176 MR1909249

[4] M Culler, C M Gordon, J Luecke, P B Shalen, Dehn surgery on knots, Ann. of Math. (2) 125 (1987) 237-300 MR881270

[5] J C Dean, Small Seifert-fibered Dehn surgery on hyperbolic knots, Algebr. Geom. Topol. 3 (2003) 435-472 MR1997325

[6] C Delman, Constructing essential laminations which survive all Dehn surgeries, preprint

[7] C Delman, Essential laminations and Dehn surgery on 2-bridge knots, Topology Appl. 63 (1995) 201-221 MR1334307

[8] C Delman, R Roberts, Alternating knots satisfy Strong Property P, Comment. Math. Helv. 74 (1999) 376-397 MR1710698

[9] D Futer, M Ishikawa, Y Kabaya, T W Mattman, K Shimokawa, Finite surgeries on three-tangle pretzel knots, Algebr. Geom. Topol. 9 (2009) 743-771

[10] D Gabai, Detecting fibred links in $S^{3}$, Comment. Math. Helv. 61 (1986) 519-555 MR870705

[11] D Gabai, U Oertel, Essential laminations in 3-manifolds, Ann. of Math. (2) 130 (1989) 41-73 MR1005607

[12] A Kawauchi, A survey of knot theory, Birkhäuser Verlag, Basel (1996) MR1417494 Translated and revised from the 1990 Japanese original by the author

[13] R Kirby, editor, Problems in low-dimensional topology, from: "Geometric topology (Athens, GA, 1993)”, AMS/IP Stud. Adv. Math. 2, Amer. Math. Soc. (1997) 35-473 MR1470751 
[14] W B R Lickorish, An introduction to knot theory, Graduate Texts in Math. 175, Springer, New York (1997) MR1472978

[15] T W Mattman, Cyclic and finite surgeries on pretzel knots, from: "Knots 2000 Korea, Vol. 3 (Yongpyong)”, J. Knot Theory Ramifications 11 (2002) 891-902 MR1936241

[16] W Menasco, Closed incompressible surfaces in alternating knot and link complements, Topology 23 (1984) 37-44 MR721450

[17] L Moser, Elementary surgery along a torus knot, Pacific J. Math. 38 (1971) 737-745 MR0383406

[18] Y Ni, Knot Floer homology detects fibred knots, Invent. Math. 170 (2007) 577-608 MR2357503

[19] U Oertel, Closed incompressible surfaces in complements of star links, Pacific J. Math. 111 (1984) 209-230 MR732067

[20] P Ozsváth, Z Szabó, Knot Floer homology and rational surgeries arXiv: math.GT/05044404

[21] P Ozsváth, Z Szabó, Holomorphic disks and three-manifold invariants: properties and applications, Ann. of Math. (2) 159 (2004) 1159-1245 MR2113020

[22] P Ozsváth, Z Szabó, On knot Floer homology and lens space surgeries, Topology 44 (2005) 1281-1300 MR2168576

[23] W P Thurston, The geometry and topology of three-manifolds, Princeton Univ. Math. Dept. Lecture Notes (1979) Available at http://msri.org/publications/books/ gt $3 \mathrm{~m} /$

[24] L Watson, Surgery obstructions from Khovanov homology arXiv:0807.1341

[25] Y-Q Wu, Dehn surgery on arborescent knots, J. Differential Geom. 43 (1996) 171-197 MR1424423

[26] Y-Q Wu, Dehn surgery on arborescent knots and links-a survey, from: "Knot theory and its applications", (C Adams, editor), Chaos Solitons Fractals 9, Elsevier, Oxford (1998) 671-679 MR1628749

School of Mathematics Education, Nara University of Education Takabatake-cho, Nara 630-8528, Japan

Graduate School of Science, Osaka City University

Osaka 558-8585, Japan

ichihara@nara-edu.ac.jp, jong@sci.osaka-cu.ac.jp

http://mailsrv.nara-edu.ac.jp/ ichihara/index.html, http://www.ex.media.osaka-cu.ac.jp/ d07sa009/index.html

Received: 2 November 2008 Revised: 25 March 2009 